\title{
On products of quartic polynomials over consecutive indices which are perfect squares
}

\section{Kantaphon Kuhapatanakul, Natnicha Meeboomak and Kanyarat Thongsing}

Department of Mathematics, Faculty of Science, Kasetsart University, Bangkok, Thailand e-mails: fscikpkk@ku.ac.th,m.nutnicha1994@hotmail.com, kanyarat.thoneku.th

Received: 31 October 2017

Accepted: 17 August 2018

Abstract: Let $a$ be a positive integer. We study the Diophantine equation

$$
\prod_{k=1}^{n}\left(a^{2} k^{4}+\left(2 a-a^{2}\right) k^{2}+1\right)=y^{2} .
$$

This Diophantine equation generalizes a result of Gürel [5] for $a=2$. We also prove that the product $\left(2^{2}-1\right)\left(3^{2}-1\right) \ldots\left(n^{2}-1\right)$ is a perfect square only for the values $n$ for which the triangular number $T_{n}$ is a perfect square.

Keywords: Diophantine equation, Perfect square, Quartic polynomial, Quadratic polynomial. 2010 Mathematics Subject Classification: 11D25, $11 \mathrm{D} 09$.

\section{Introduction}

The study of sequences containing infinitely many squares is a common topic in number theory. Let $\Omega_{\mu}(n)=\left(1^{\mu}+1\right)\left(2^{\mu}+1\right) \ldots\left(n^{\mu}+1\right)$ where $\mu \geq 2$ is an integer. Amdeberhan et al. [1] conjectured that $\Omega_{2}(n)$ is not a square for any integer $n>3$. Cilleruelo [3] confirmed this conjecture. Gürel and Kisisel [6] proved that $\Omega_{3}(n)$ is not a square. Later, an idea due to Zudilin was applied to $\Omega_{p}(n)$ by Zhang and Wang [9] and to $\Omega_{p^{t}}(n)$ by Chen et al. [2] for any odd prime $p$. Fang [4] confirmed another similar conjecture posed by Amdeberhan et al. [1] to the 
products of quadratic polynomials $\prod_{k=1}^{n}\left(4 k^{2}+1\right)$ and $\prod_{k=1}^{n}\left(2 k^{2}-2 k+1\right)$ are not perfect squares. Yang et al. [8] studied the Diophantine equation $\prod_{k=1}^{n}\left(a k^{2}+b k+c\right)=d y^{l}$. Gürel [5] proved that the product $\prod_{k=1}^{n}\left(4 k^{4}+1\right)$ is a perfect square for infinitely many $n$.

In this manuscript, we will extend the result of Gürel [5] on the polynomial $4 k^{4}+1$ to the polynomial $P_{a}(k)=a^{2} k^{4}+\left(2 a-a^{2}\right) k^{2}+1$, where $a$ is a positive integer. Next, we prove the product $\left(2^{2}-1\right)\left(3^{2}-1\right) \ldots\left(n^{2}-1\right)$ is a perfect square only for the values $n$ for which the triangular number $T_{n}$ is a perfect square.

\section{Main results}

Let $a$ be a positive integer and $P_{a}(x)=a^{2} x^{4}+\left(2 a-a^{2}\right) x^{2}+1$. Denote $\mathcal{X}_{a}(n)$ is the product of first $n$ consecutive values of the $P_{a}(n)$, i.e.,

$$
\mathcal{X}_{a}(n)=P_{a}(1) P_{a}(2) \ldots P_{a}(n)
$$

Lemma 1. $\mathcal{X}_{a}(n)$ is a square if and only if $a n^{2}+a n+1$ is a square.

Proof. Let $f(x)=a x^{2}-a x+1$. Then $f(x+1)=a x^{2}+a x+1$ and

$$
P_{a}(x)=a^{2} x^{4}+\left(2 a-a^{2}\right) x^{2}+1=\left(a x^{2}-a x+1\right)\left(a x^{2}+a x+1\right)=f(x) f(x+1) .
$$

We have

$$
\mathcal{X}_{a}(n)=\prod_{k=1}^{n} P_{a}(k)=\prod_{k=1}^{n} f(k) f(k+1)=\left(\prod_{k=2}^{n} f(k)\right)^{2} f(1) f(n+1) .
$$

Since $f(1)=1$, it follows that $\mathcal{X}_{a}(n)$ is a square if and only if $f(n+1)$ is a square.

Consider $4 n^{2}+4 n+1=(2 n+1)^{2}$, we obtain that

$$
\prod_{k=1}^{n}\left(16 k^{4}-8 k^{2}+1\right) \text { is a perfect square for all } n \text {. }
$$

Theorem 1. Let $a, d, n$ be positive integers with $a=d^{2} \neq 4$. Suppose $p=\left\lfloor\frac{d+1}{2}\right\rfloor$. Then $\mathcal{X}_{a}(n)$ is not a perfect square for $n>\frac{p^{2}-2 p}{d^{2}-2 d p+2 d}$.

Proof. By Lemma 1, the problem is reduced to finding square values of $f(n+1)$, i.e., finding integer solutions to the following equation,

$$
a n^{2}+a n+1=m^{2} .
$$

We see that

$$
a n^{2}+a n+1=d^{2} n^{2}+d^{2} n+1<(d n+p)^{2} .
$$

Assume $n>\left(p^{2}-2 p\right) /\left(d^{2}-2 d p+2 d\right)$. 
If $d$ is even, we get that $p=\frac{d}{2}$ and $n>\frac{d-4}{8}$, so

$$
d^{2} n^{2}+d^{2} n+1>(d n+p-1)^{2} .
$$

And if $d$ is odd, we have that $p=(d+1) / 2$ and $n>\left(d^{2}-2 d-3\right) / 4 d$, so

$$
d^{2} n^{2}+d^{2} n+1>(d n+p-1)^{2} .
$$

We obtain that

$$
(d n+p-1)^{2}<d^{2} n^{2}+d^{2} n+1<(d n+p)^{2} .
$$

Since there is no perfect square between two consecutive perfect squares, $\mathcal{X}_{a}(n)$ is not a perfect square for $n>\frac{p^{2}-2 p}{d^{2}-2 d p+2 d}$ and $a=d^{2} \neq 4$.

For $a$ not a perfect square, we conjecture that the Diophantine equation (1) has infinitely many solutions. The case $a=2$ has been shown in [5]. In the next theorem, we will only show the case $3 \leq a \leq 13$.

Theorem 2. Let $3 \leq a \leq 13$ be not a perfect square. Then $\mathcal{X}_{a}(n)$ is a perfect square for infinitely many $n$.

Proof. It suffices to find the integer solutions of (1).

- Case $a=3$, we consider

$$
3 n^{2}+3 n+1=m^{2} .
$$

We see that $(7,13)$ is a solution of (2). For each solution $(x, y)$ of (2), the map sends $(x, y)$ to $(7 x+4 y+3,12 x+7 y+6)$, which gives another solution of (2). It can be verified that

$$
\begin{aligned}
3(7 x+4 y+3)^{2}+3(7 x+4 y+3)+1 & =147 x^{2}+48 y^{2}+168 x y+147 x+84 y+37 \\
& =\left(3 x^{2}+3 x+1\right)-y^{2}+(12 x+7 y+6)^{2} \\
& =(12 x+7 y+6)^{2} .
\end{aligned}
$$

Therefore, the equation (2) has infinitely many distinct solutions.

(Note: If $\left(n_{i}, m_{i}\right)$ are all solutions of (2), the sequence $\left\{n_{i}\right\}$ satisfies $n_{i}=14 n_{i-1}-n_{i-2}+6$, where $n_{0}=0, n_{1}=7$, see A001921 in [7], and the sequence $\left\{m_{i}\right\}$ is A001570 in [7].)

- Case $a=5$, we consider

$$
5 n^{2}+5 n+1=m^{2} .
$$

Clearly, $(8,19)$ is a solution of (3). For each solution $(x, y)$ of $(3)$, the map sends $(x, y)$ to $(9 x+4 y+4,20 x+9 y+10)$, which gives another solution of (3).

(Note: If $\left(n_{i}, m_{i}\right)$ are all solutions of $(3)$, then $n_{i}=\left(F_{6 i+3}-2\right) / 4$ and $m_{i}=\left(F_{6 n+4}+\right.$ $\left.F_{6 n+2}\right) / 4$, where $F_{i}$ is $i^{\text {th }}$ Fibonacci number, see $A 053606$ and A049629 in [7].) 
- Case $a=6$, we consider

$$
6 n^{2}+6 n+1=m^{2}
$$

Clearly, $(4,11)$ is a solution of (4). For each solution $(x, y)$ of $(4)$, the map sends $(x, y)$ to $(5 x+2 y+2,12 x+5 y+6)$, which gives another solution of (4).

(Note: If $\left(n_{i}, m_{i}\right)$ are all solutions of $(4)$, then $n_{i}=11 n_{i-1}-11 n_{i-2}+n_{i-3}$, where $n_{0}=0$, $n_{1}=4$ and $n_{2}=44$, see A105038 in [7] and $m_{i}$ is A054320 in [7].)

- Case $a=7$, we consider

$$
7 n^{2}+7 n+1=m^{2}
$$

Clearly, $(15,41)$ is a solution of $(5)$. For each solution $(x, y)$ of $(5)$, the map sends $(x, y)$ to $(127 x+48 y+63,336 x+127 y+168)$, which gives another solution of (5).

(Note: If $\left(n_{i}, m_{i}\right)$ are all solutions of (5), then $n_{i}=254 n_{i-2}-n_{i-4}+126$, where $n_{0}=0$, $n_{1}=15, n_{2}=111$ and $n_{3}=3936$, see A105051 or A105040 in [7].)

- Case $a=8$, we consider

$$
8 n^{2}+8 n+1=m^{2}
$$

Clearly, $(2,7)$ is a solution of (6). For each solution $(x, y)$ of $(6)$, the map sends $(x, y)$ to $(3 x+y+1,8 x+3 y+4)$, which gives another solution of (6).

(Note: If $\left(n_{i}, m_{i}\right)$ are all solutions of (6), the sequences $\left\{n_{i}\right\}$ and $\left\{m_{i}\right\}$ are respectively A053141 and A002315 in [7].)

- Case $a=10$, we consider

$$
10 n^{2}+10 n+1=m^{2} .
$$

Clearly, $(3,11)$ is a solution of $(7)$. For each solution $(x, y)$ of $(7)$, the map sends $(x, y)$ to $(19 x+6 y+9,60 x+19 y+30)$, which gives another solution of (7).

(Note: If $\left(n_{i}, m_{i}\right)$ are all solutions of (7), the sequence $\left\{n_{i}\right\}$ is A222390 in [7].)

- Case $a=11$, we consider

$$
11 n^{2}+11 n+1=m^{2} .
$$

Clearly, $(39,131)$ is a solution of $(8)$. For each solution $(x, y)$ of $(8)$, the map sends $(x, y)$ to $(199 x+60 y+99,660 x+199 y+330)$, which gives another solution of $(8)$.

(Note: If $\left(n_{i}, m_{i}\right)$ are all solutions of (8), the sequences $\left\{n_{i}\right\}$ and $\left\{m_{i}\right\}$ are respectively A105838 and A105837 in [7].)

- Case $a=12$, we consider

$$
12 n^{2}+12 n+1=m^{2} .
$$

Clearly, $(1,5)$ is a solution of (9). For each solution $(x, y)$ of (9), the map sends $(x, y)$ to $(7 x+2 y+3,24 x+7 y+12)$, which gives another solution of (9).

(Note: If $\left(n_{i}, m_{i}\right)$ are all solutions of (9), the sequences $\left\{n_{i}\right\}$ and $\left\{m_{i}\right\}$ are respectively A061278 and A001834 in [7].) 
- Case $a=13$, we consider

$$
13 n^{2}+13 n+1=m^{2} .
$$

Clearly, $(7,27)$ is a solution of $(10)$. For each solution $(x, y)$ of $(10)$, the map sends $(x, y)$ to $(649 x+180 y+324,234 x+649 y+1170)$, which gives another solution of (10).

(Note: If $\left(n_{i}, m_{i}\right)$ are all solutions of (10), the sequence $\left\{n_{i}\right\}$ is A104240 in [7].)

Therefore, $\mathcal{X}_{a}(n)$ is a perfect square for infinitely many $n$, where $a$ is not a perfect square.

For $a>13$ not a perfect square, the authors consider that $\mathcal{X}_{a}(n)$ is a perfect square for infinitely many $n$. We can find the linear map for each solution $\left(n_{0}, m_{0}\right)$ that gives another solution of (1). But the authors will leave this problem to the interested reader.

From Theorems 1 and 2, we give the following examples for $a=1,3,5$.

(1) $\prod_{k=1}^{n}\left(k^{4}+k^{2}+1\right)$ is not a square.

(2) $\prod_{k=1}^{n}\left(9 k^{4}-3 k^{2}+1\right)$ and $\prod_{k=1}^{n}\left(25 k^{4}-15 k^{2}+1\right)$ are perfect squares for infinitely many $n$.

Next, we give analogue of $\Omega_{2}(n)$ for the product $\left(2^{2}-1\right)\left(3^{2}-1\right) \ldots\left(n^{2}-1\right)$.

Theorem 3. The product $\prod_{k=2}^{n}\left(k^{2}-1\right)$ is a perfect square if and only if the triangular number $T_{n}$ is a perfect square for $n>1$.

Proof. The triangular number $T_{n}$ is a number obtained by adding all positive integers less than or equal to a given positive integer $n$, i.e., $T_{n}=\sum_{i=1}^{n} i=\frac{n(n+1)}{2}$. We have

$$
\begin{aligned}
\prod_{k=2}^{n}\left(k^{2}-1\right) & =\prod_{k=2}^{n}(k-1)(k+1) \\
& =\left(\prod_{k=3}^{n-1} k\right)^{2} 2 n(n+1) \\
& =\left(\prod_{k=3}^{n-1} k\right)^{2} 4 T_{n} .
\end{aligned}
$$

Thus, this product is a square if and only if $T_{n}$ is a square.

The triangular number $T_{n}$ is a square (see $A 001108$ in [7]) when the value of $n$ is $1,8,49,288$, $1681,9800,57121,332928,1940449,11309768, \ldots$.

\section{Acknowledgements}

The authors would like to thank the anonymous referee for his/her helpful suggestions. This work is supported by Department of Mathematics, Faculty of Science, Kasetsart University, Thailand. 


\section{References}

[1] Amdeberhan, T., Medina, L. A., \& Moll, V. H. (2008) Arithmetical properties of a sequence arising from an arctangent sum, J. Number Theory, 128(6), 1807-1846.

[2] Chen, Y. G., Gong, M. L., \& Ren, X. Z. (2013) On the products $\left(1^{l}+1\right)\left(2^{l}+1\right) \ldots\left(n^{l}+1\right)$, J. Number Theory, 133(8), 2470-2474.

[3] Cilleruelo, J. (2008) Squares in $\left(1^{2}+1\right) \ldots\left(n^{2}+1\right)$, J. Number Theory, 128(8), 2488-2491.

[4] Fang, J. H. (2009) Neither $\prod_{k=1}^{n}\left(4 k^{2}+1\right)$ nor $\prod_{k=1}^{n}(2 k(k-1)+1)$ is a perfect square, Integers, 9, 177-180.

[5] Gürel, E. (2016) On the occurrence of perfect squares among values of certain polynomial products, Amer. Math. Monthly, 123(6), 597-599.

[6] Gürel, E. \& Kisisel, A. U. O. (2010) A note on the products $\left(1^{\mu}+1\right) \ldots\left(n^{\mu}+1\right)$, J. Number Theory, 130(1), 187-191.

[7] Sloane, N. J. A. (2011) The On-Line Encyclopedia of Integer Sequences. Published electronically at http://oeis.org.

[8] Yang, S., Togbé, A. \& He, B. (2011) Diophantine equations with products of consecutive values of a quadratic polynomial, J. Number Theory, 131(5), 1840-1851.

[9] Zhang, W. \& Wang, T. (2012) Powerful numbers in $\left(1^{k}+1\right)\left(2^{k}+1\right) \ldots\left(n^{k}+1\right)$, J. Number Theory, 132(11), 2630-2635. 